Recepción: 18 / 05 / 2018

Aceptación: 29 / 07 / 2018

Publicación: 05 / 10 / 2018

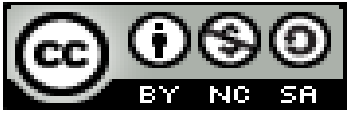

Ciencias de la educación Artículo de investigación

\title{
Incidencia de la educación especial en el rendimiento escolar en los estudiantes de educación básica
}

Incidence of special education on school performance in basic education students

\section{Incidência de educação especial sobre o desempenho escolar em estudantes de educação básica}

\author{
Lidia A. Freire-Ramos ${ }^{\text {I }}$ \\ lidiaalexafreire84@outlook.com \\ Leandro F. Gallegos-Gallegos II \\ faby01gallegos@hotmail.com
}

\section{Correspondencia: lidiaalexafreire84@outlook.com}

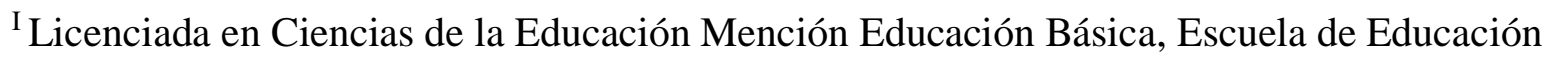
Básica Abdón Calderón, Guayaquil, Ecuador.

II Ingeniero en Contabilidad y Auditoría CPA, Tecnólogo en Análisis de Sistemas, Unidad Educativa Rosa Zarate, Quito, Ecuador. 


\section{Resumen}

La organización y clima familiar constituyen mecanismos esenciales en la formación de la persona, especialmente en los primeros años de vida. El ser humano encuentra en la familia, los primeros modos de vida, que permiten el desarrollo de las diversas dimensiones de la persona. En las últimas décadas, se han relacionado el ambiente familiar con el rendimiento académico, siendo una de las grandes hipótesis, que: Un buen clima familiar, es fruto de una estructura estable y adecuada, asegura un buen desarrollo de la persona, y por ende un buen desempeño académico. Entonces podemos concluir que los valores en la disciplina son trascendentales primero en las personas y después en la sociedad, ya que de este orden dependerá que los valores se conviertan parte de la formación integral de los estudiantes.

Palabras clave: disciplina; normas; família; valores; rendimento.

\section{Abstract}

Discipline is the orderly and methodical way of doing things, following a set of strict rules that, usually, govern an activity. Family organization and climate are essential mechanisms in the formation of the person, especially in the first years of life. The human being finds in the family, the first ways of life, which allow the development of the various dimensions of the person. In the last decades, the family environment has been related to academic performance, being one of the great hypotheses that: A good family climate is the result of a stable and adequate structure, ensures a good development of the person, and therefore a good academic performance. Then we can conclude that the values in the discipline are transcendental first in the people and later in the society, since of this order will depend that the values become part of the integral formation of the students.

Key words: discipline; standards; family; values; performance.

\section{Resumo}

A organização familiar e o clima são mecanismos essenciais na formação da pessoa, principalmente nos primeiros anos de vida. O ser humano encontra na família, os primeiros modos de vida, que permitem o desenvolvimento das diferentes dimensões da pessoa. Nas últimas décadas, o ambiente familiar tem sido relacionado ao desempenho acadêmico, uma das 
grandes hipóteses é que: Um bom clima familiar é o resultado de uma estrutura estável e adequada, garante um bom desenvolvimento da pessoa e, portanto, bom desempenho acadêmico. Então podemos concluir que os valores na disciplina são transcendentais primeiro na pessoa e depois na sociedade, já que nesta ordem dependerá que os valores se tornem parte da formação integral dos alunos.

Palavras chave: disciplina; normas; família; valores; desempenho.

\section{Introducción}

Disciplina es un tema muy importante estamos viviendo una época donde los valores humanos están siendo menospreciados, y ahora cualquier persona que hable sobre valores, la considera anticuada o pasada de moda.

Ahora los valores los estamos adoptando a nuestra nueva forma de vivir y esta sociedad cada día más liberalista, hoy en día lo que está de moda, son aquellos valores que nos permitan ser libres, y que nos ayuden a evadir responsabilidades.

Es por eso que debemos tener conciencia de que todavía estamos a tiempo para que nuestros pequeños tengan una buena guía por parte de nosotros que estamos viendo a que grado han llegado las cosas, es muy importante que desde niños se le valla inculcando y enseñando que hay herramientas valiosas que tiene el ser humano para llevar una vida digna y en armonía, que los preparemos para lo que se avecina, para que se les pueda dar una buena preparación sobre disciplina, no solo deben intervenir los padres, también juegan un papel muy importante los maestros de ellos depende la disciplina dentro del aula, educar para la vida y por la vida.

Entonces podemos concluir que los valores en la disciplina son trascendentales primero en las personas y después en la sociedad, ya que de este orden dependerá que los valores se conviertan parte de la formación integral de los estudiantes. 


\section{Introducción al problema}

La convivencia dentro de las aulas se basa en los derechos de cada uno de los estudiantes, pero también por las obligaciones y responsabilidades que tienen y deben ser asumidas, como las normas de comportamiento. (Fuentes, 2015)

Sin reglas o normas que guíen el comportamiento de los estudiantes, pueden ser más liberales y hacer lo que ellos quisieran y pensarían que ese actuar es correcto, el aula de clases sencillamente se convertiría en un escenario de discordia, de falta de valores y de irrespeto por los demás. (Fuentes, 2015)

Las reglas o normas dentro del aula, admiten que se tenga un ambiente solidario, donde el respeto es la base de la comunicación y la interacción entre los miembros del grupo. (Fuentes, 2015)

Establecer normas dentro del aula, es primordial para llevar una sana convivencia y los objetivos y las metas propuestas en cuanto a enseñanza se puedan conseguir de forma satisfactoria. (Fuentes, 2015)

\section{Normas de comportamiento en el aula}

Como se mencionó en el principio, debe tener claro que las normas de comportamiento pueden cambiar dependiendo del curso educativo en el que se encuentre, pero hay ciertas normas básicas que deben ser implementadas y seguidas en todas las aulas de clase. (Fuentes, 2015)

Se debe ingresar y salir de forma ordenada al aula de clase.

Se debe saludar y despedirse

Debe esperar su turno para tomar la palabra en la clase.

Se debe poner atención durante la clase.

Se debe escuchar a los demás compañeros.

Los estudiantes deben sentar correctamente. 
Se debe escuchar y atender a los docentes.

Ayudar a sus compañeros si lo requiere.

Hablar en voz baja.

No correr dentro del aula.

Cuidar el material en el aula.

Ser puntual a la hora de llegar a clase.

Ser ordenado.

No interrumpir cuando otra persona está hablando.

Las anteriores son las normas básicas que todo alumno debe cumplir, sin embargo, el maestro debe también seguir unas normas básicas dentro del aula:

Empezar las clases de forma puntual.

Ser respetuoso con los alumnos.

Tener el material adecuado para impartir las lecciones.

Hablar de forma clara y precisa.

Utilizar un tono de voz moderado.

Ser imparcial con los alumnos.

Tratar a todos sus estudiantes por igual.

Debe saber que cada Institución Educativa puede implementar sus propias normas de comportamiento según los valores éticos y el tipo de formación que este implemente, sin embargo, siempre debe imponerse el respeto frente a los demás, la tolerancia, la igualdad de derechos y la libertad de expresión. (Fuentes, 2015) 
Siguiendo pautas y normas es como se fortalece una sociedad, es por eso que la implementación estas desde pequeños escenarios como el aula de clases, permite que se formen adultos mas éticos. (Fuentes, 2015).

\section{Disciplina}

La disciplina es un modelo a seguir, son reglas establecidas para llevar un comportamiento adecuado para consigo y los demás dentro de una sociedad. Con estas reglas el individuo sabe la manera de comportarse y de esta manera llevar una vida llena de armonía y servir de ejemplo para quienes lo rodean, logrando ser un ente positivo en este mundo exigente.

Los valores van de la mano para que esto de éxito y concluya dicha formación de seres humanos más flexibles, comprensivos y educados. El ser humano desde su nacimiento nace aprendiendo y la disciplina lo aplicamos desde que nacemos con la ayuda de sus padres a quienes imitamos según sus valores y principios.

La disciplina conductista se concibe como un conjunto de programas disciplinarios o como una política de disciplina en el centro educativo. Es cierto que un conjunto de programas disciplinarios puede prevenir y controlar los problemas de conductas de los alumnos coordinando los procedimientos disciplinarios establecidos en el centro e informando a los alumnos acerca de las que se esperan de ellos y cuales se consideran prohibidas. (García Correa, 2015)

\section{La disciplina (tarde o temprano) vencerá a la inteligencia}

La disciplina es lo que nos hace puntuales y de esta forma respetamos nuestro tiempo y el de los demás.

La disciplina es lo que hace que repasemos la calidad de nuestro trabajo a pesar de que nos sintamos cansados y así evitamos errores o trabajos mediocres.

La disciplina hace que nos entrenemos aún más fuerte después de una derrota y consigue que la derrota sirva para hacernos más fuertes. 
La disciplina es lo que hace que nos levantemos más temprano para ir a hacer deporte y así tener mejor energía y sentirnos mejor en nuestra casa y en nuestro trabajo. (Kenji, 2014)

El término acoso (bullying) hace referencia a un comportamiento repetitivo de hostigamiento e intimidación, cuyas consecuencias suelen ser el aislamiento y la exclusión social de la víctima

\section{Hablamos de acoso cuando se cumplen al menos tres de los siguientes criterios:}

La víctima se siente intimidada.

La víctima se siente excluida.

La víctima percibe al agresor como más fuerte

Las agresiones son cada vez de mayor intensidad

Las agresiones suelen ocurrir en privado.

El acoso va acompañado de una "ley del silencio"

ÍNCIVICO. El que no respeta y vive las normas de la sociedad

\section{Estilos de disciplina parental}

Los estilos parentales y se manifiestan a sí mismos en las habilidades de los padres para equilibrar la disciplina, como crear y reforzar los límites con cariño o responder a las necesidades de amor, afecto y afirmación del niño. Conforme buscas proporcionar a tu hijo una base estable para la vida a lo largo de su infancia, entender estas técnicas de disciplina básica e identificar la que ajusta contigo, te puede ayudar a ajustar su estilo parental para darle a tu hijo el mejor comienzo posible en la vida. (Muy fitness, 2016)

\section{Positivo}

De acuerdo con la Universidad de Wisconsin, los padres positivos son aquellos que tienen un buen equilibrio entre la disciplina y el cariño, y tratan de ofrecer una cantidad equitativa de los dos. Algunas veces son llamados padres "con autoridad" o "democráticos". Tienen altas

\section{4}

Pol. Con. (Edición núm. 26) Vol. 3, No 10, octubre 2018, pp. 58-74, ISSN: 2550 - 682X 
expectativas sobre sus hijos y refuerzan sus reglas consistentemente, aunque también se comunican con sus hijos y están dispuestos a escuchar. Estos niños se sienten amados y respetados, pero entienden que necesitan seguir las reglas de la familia. También tienden a tener éxito en la escuela, bajos niveles de ansiedad y una autoconfianza alta, dice Joan E. LeFebvre, una agente de vida familiar en la Universidad. (Muy fitness, 2016)

\section{Permisivo}

Los padres permisivos también pueden crear hijos con una autoestima alta y bajos niveles de ansiedad, de acuerdo con LeFebvre. Estos padres dependen mucho del lado del "cuidado" del espectro. Muestran mucho amor y apoyan a sus hijos, pero les falta disciplina y estructura. Algunas veces son llamados padres "indulgentes" y tienen una filosofía muy centrada en los hijos. Los niños cuyos padres no establecen límites claros frecuentemente se vuelven irrespetuosos e incapaces de tomar sus propias decisiones, advierte KeepKidsHealthy.com. (Muy fitness, 2016)

\section{Dominante}

Los padres dominantes tienden a depender mucho del aspecto de las "reglas" de las cosas, pasando por alto el lado del cuidado. Estos padres valoran la obediencia y la demandan a sus hijos, pero no los alientan a pensar o actuar de forma independiente. Tampoco permiten que su autoridad sea cuestionada. Los niños que tienen padres dominantes pueden tener pocos problemas de comportamiento, pero también exhiben grandes niveles de ansiedad y autoestima baja, dice LeFebvre. Estos padres algunas veces son llamados "autoritarios". (Muy fitness, 2016)

\section{Desvinculados}

Los padres desvinculados están completamente centrados en ellos. No nutren o disciplinan a sus hijos. Se hacen cargo de las necesidades físicas básicas, como la alimentación y la ropa, pero de otra forma no les dan mucha atención. Estos padres pueden ser considerados negligentes. Los niños con padres desvinculados tienen baja autoestima, altos niveles de ansiedad, muchos problemas de comportamiento y pobre logro académico, de acuerdo con LeFebvre. (muy fitness , 2016) 


\section{Valores}

Los valores surgen con un especial significado y cambian o desaparecen en las distintas épocas. Es precisamente el significado social que se atribuyen a los valores uno de los factores que influyen para diferenciar los valores tradicionales, aquellos que guiaron a la sociedad en el pasado, generalmente referidos a costumbres culturales o principios religiosos, y los valores modernos, compartidos por las personas en la actualidad. (rena.edu.ve, 2012)

\section{La Indisciplina}

La indisciplina trae consigo muchas causas en el alumno, una serie de hechos indisciplinarías tienen su origen en los propios alumnos, pueden ser de carácter biológico, social, y psicológico, en el profesor, mala dirección del curso, empleó constante de la misma técnica de enseñanza, conocimiento deficiente de la asignatura, acción aislada de los profesores, impuntualidad, personalidad desajustada, tipo vanidoso y presumido, tipo tímido y desconfiado etc. En la escuela se da la indiscipline por cursos numerosos, preparación deficiente de los directores, ambiente pedagógico inadecuado, abandono de los profesores a los alumnos y en la sociedad estímulos negativos, radicaciones ideológicas, omisión de los responsables de la educación. (Mayer 1998)

\section{Causas de la Indisciplina}

El estudio de las causas de la indisciplina es de vital importancia, toda vez que son variadas y complejas, se pueden originar en el hogar, en la sociedad misma, en el propio docente estudiante. Los padres viven en el mundo del trabajo y sus hijos están al cuidado de otras personas, se concentran a ver programas de televisión de violencia, pasan horas enteras en el internet viendo películas y programas de drogas, crímenes, de sexo, no hay contra de sus padres en la sociedad han perdido los valores como el respeto, la cooperación, la solidaridad, el buen ejemplo, la religiosidad respectivamente. El docente es impuntual, no planifica sus clases por lo tanto improvisa los temas de clase, mantiene la distancia entre estudiante y docente, no brinda confianza, no da oportunidades de recuperar sus calificaciones con otras tareas. Los estudiantes que no cumplen con las tareas ponen el desorden en clases, son agresivos, irrespetuosos. Por lo que se debe buscar estrategias y normas disciplinares que superen este problema que perjudica al buen aprendizaje de los estudiantes. 
El Dr. Albuja en su programa televisivo brinda claras los días domingos sobre los múltiples problemas de la indisciplina, puntualizando la agresividad, la irresponsabilidad, impuntualidad, tienen como consecuencia un bajo rendimiento académico.

Se puntualiza la indisciplina como problema al que debemos buscar estrategias de solución y hacer las respectivas recomendaciones para orientar de mejor manera las actividades académicas dentro del aula de clases. (Albuja, 2016)

\section{Importancia del problema}

La presente investigación que se ha venido investigando con firman que la disciplina sique siendo un problema global dentro de esta sociedad cada vez más exigente en el liberalismo. Y el problema persiste desde tiernas edades (Chris Watkins, Patsy Wagnerk, 2016)

Los valores ayudan a crecer y hacen posible el desarrollo agradable de todas las cualidades que tiene el ser humano y, desde la escuela se han manifestado acciones que afectan a la conducta que configuran y modelan ideas, sentimientos, actuaciones dentro del ambiente familiar, escolar y social ( Patricia Cabrera S, Karina Ochoa B, 2010). Los valores pueden ser identificados, a través de las reacciones afectivas y de las actitudes a que dan lugar mediante la observación de las conductas que pueden responder a un valor determinado (Patricia Cabrera S, Karina Ochoa B, 2010). La escuela constituye un elemento indispensable, pero no suficiente, para la educación en valores, presentándose como un agente intermediario entre la familia y el estado, además es un mecanismo transformador que históricamente se va adecuando de acuerdo a las necesidades del momento presente y futuro (Patricia Cabrera S, Karina Ochoa B, 2010). Estos valores hacen referencia a proyectos o metas en relación con un determinado tipo de conducta, por ejemplo, la honestidad, la responsabilidad, la igualdad, ya que los valores se presentan de forma personalizada, es decir, cambian según las circunstancias socioculturales (Patricia Cabrera S, Karina Ochoa B, 2010).

Educar a los niños y niñas en valores no significa imponer sino más bien proponer, abrir diferentes caminos y opciones, ayudar a que cada uno vea cuales son los mejores valores para ellos. Educar es ayudar a los niños y niñas a desarrollar la capacidad de elección y, a actuar de 
acuerdo a sus metas e ideales, impulsando la coherencia entre lo que piensan, dicen y hacen dentro de las aulas de clases (Patricia Cabrera S, Karina Ochoa B, 2010).

Los padres son una parte importante, no solo para el cambio y la dinamización de las nuevas tendencias educativas e integradoras, sino, sobre todo, para mejorar la situación de los ESTRATÉGIAS METODOLÓGICAS PARA PREVENIR LAS CONDUCTAS DISRUPTIVAS Dirigidas a padres Dirigidas a Profesores Dirigidas a niños y niñas Estudio del impacto de las Conductas Disruptivas en niños y niñas dentro del aula de clases (Patricia Cabrera S, Karina Ochoa B, 2010).

La disciplina es el medio, la herramienta con la que debe contar el educador para poder guiar y organizar el aprendizaje y al mismo tiempo es un fin para desarrollar en la persona los valores, actitudes que se deseen. En un primer momento debe ejercerse la disciplina externa, pero esta paulatinamente tiene que apuntar hacia la disciplina interna, la autodisciplina que es la verdadera disciplina (secchi jeremías) No podemos comentar sobre disciplina escolar si no llevamos a los educandos a la ética de valores (familiares, sociales, nacionales y sobre todo con ellos mismos) y al reconocer un mundo que también tiene aspectos positivos los lleve como respuesta a un compromiso, lleno de una sana disciplina emanada desde el interior, para un mundo mejor. (Carlos Zamarripa) No podemos comentar sobre disciplina escolar si no llevamos a los educandos a la ética de valores (familiares, sociales, nacionales y sobre todo con ellos mismos) y al reconocer un mundo que también tiene aspectos positivos los lleve como respuesta a un compromiso, lleno de una sana disciplina emanada desde el interior, para un mundo mejor. (Carlos Zamarripa) Es un tipo de relación interrumpa que nace y surge de la convivencia entre un grupo homogéneo de personas y otro grupo reducido (puede ser una sola persona) al cual se le reconoce su autoridad sin ejercer violencia alguna. (Pedro García).

\section{Metodología}

La presente investigación tiene un enfoque cualitativo y cuantitativo pues se basa en muestras reducidas, requiere de un trabajo de campo de todos los participantes, sus resultados no son generalizados y sus objetivos plantean acciones inmediatas, ya que dicha investigación posee una información que se obtiene. 
De acuerdo al problema requiere la interpretación previa a un análisis lo que me va a permitir elaborar o formular hipótesis para solucionar el problema.

Este trabajo investigativo se ha desarrollado basándonos en la disciplina y enfocándonos en el rendimiento escolar dentro del cual están involucrados estudiantes, profesores, buscando solucionar problemas encontrados a lo largo de nuestra investigación.

\section{Modalidad Básica de la Investigación}

Para la información de la presente investigación se utilizó dos tipos de investigación como son bibliográficas o documentales y de campo.

\section{Resultados}

Esta investigación se enmarca en una perspectiva empírico-analítica de carácter cuantitativo. Se trata de un estudio descriptivo y de contraste de variables entre dos grupos de datos independientes: profesores y alumnos.

Las percepciones analizadas en ambos grupos corresponden a las formas de intervención o sanciones percibidas, asociadas a determinados comportamientos disruptivos que tienen lugar en el aula.

\section{Participantes}

La muestra de estudio está compuesta por 57 estudiantes y 3 docentes

\section{Instrumentos}

El instrumento utilizado para la recogida de datos fue un cuestionario, elaborado para tal fin, dirigido a obtener las percepciones de los profesores y las de los estudiantes sobre disciplina escolar.

Se confeccionó una versión para profesores y otra para alumnos que diferían en el tipo de lenguaje empleado para describir las situaciones-estímulo, así como en el tratamiento otorgado a los sujetos encuestados. Ambas versiones del cuestionario incluían los siguientes apartados: 
Un apartado introductorio, con indicación del objetivo del cuestionario y de las principales orientaciones para complementarlo.

Una tabla de doble entrada en la que se solicitaba a los sujetos encuestados que seleccionaran para cada mal comportamiento sugerido, el tipo o tipos de intervención que utilizaban (en el caso de los profesores) u observaban utilizar (en el de los estudiantes) a fin de interrumpir la actuación disruptiva.

Los comportamientos presentados, así como las formas de intervención, se basaron en los datos más significativos obtenidos al respecto en estudios anteriores (Añaños y Gotzens, 1990; Martin, Linfoot y Stepheson, 1999).

La validez del instrumento se sometió a la Prueba de Jueces, considerando los criterios de comprensibilidad, pertinencia e importancia, mientras que el análisis de la fiabilidad se llevó a cabo en un estudio piloto realizado con 3 profesores y 57 estudiantes, utilizando las técnicas de Test-retest y alfa de Cronbach. Los resultados obtenidos aportaron valores de 0,8721 y 0,9064, así como 0,8542 y 0,8879 para profesores y estudiantes respectivamente, en cada una de las pruebas citadas, los cuales pueden considerarse como muy satisfactorios y dan fe de la correcta fiabilidad del cuestionario.

\section{Resultados}

La explotación estadística de los resultados se llevó a cabo mediante la prueba de Chi-cuadrado de Pearson con corrección de Yates, en la medida en que se manejaban variables categóricas. Se aplicó el estadístico exacto de Fisher en los casos en los que los efectivos eran inferiores a cinco.

A grandes rasgos, el análisis de los resultados ha dado lugar a la verificación de nuestra hipótesis, ya que tal como recoge la Tabla 2 se observan diferencias significativas entre las percepciones de profesores y estudiantes, tanto en el tipo de comportamientos que profesores y estudiantes dicen ser castigados, como en las estrategias disciplinarias que dicen utilizar los profesores y las que dicen recibir o constatar los estudiantes.

En cuanto al número de comportamientos que merecen ser sancionados en el aula, hemos constatado diferencias significativas de los 22 ejemplos de malos comportamientos presentados en el cuestionario, siendo los profesores quienes declaran hacer uso de castigos a los 
comportamientos disruptivos presentados en el cuestionario con mayor frecuencia que los estudiantes dicen percibir. Sólo en dos casos («utilizar el móvil» y «mascar chicle los estudiantes perciben mayor abundancia de castigos que los profesores dicen aplicar. Así pues, en términos generales, son los profesores quienes más comportamientos dicen sancionar en el aula.

Sin embargo, a pesar de las diferencias significativas observadas, la tendencia entre profesores y estudiantes en cuanto al reconocimiento de comportamientos sancionados no presenta propiamente discrepancias; en todo caso se observan diferencias relativas al grado de consenso entre los sujetos de ambos grupos, pero manteniendo una valoración relativamente consonante en lo que a interrupción de los comportamientos perturbadores se refiere.

\section{Tabla 1}

\begin{tabular}{|c|c|c|c|c|c|c|c|c|c|c|}
\hline & X (Comportamientos de los estudiantes) & $\mathbf{F}$ & $\mathbf{F}$ & $\mathrm{H}$ & $\mathbf{H} \%$ & $\mathbf{X}$ & $\mathbf{H} \%$ & $\mathbf{F}$ & $\mathbf{F}$ & $\mathbf{H}$ \\
\hline C01 & Deambulación por el aula & 2 & 2 & 0,04 & 3,57 & $\mathrm{C} 01$ & 3,57 & 2 & 2 & 0,04 \\
\hline $\mathrm{C} 02$ & Agresiones al mobiliario & 2 & 4 & 0,04 & 3,57 & $\mathrm{C} 02$ & 3,57 & 2 & 4 & 0,04 \\
\hline $\mathrm{C} 03$ & Destrucción de material escolar & 2 & 6 & 0,04 & 3,57 & $\mathrm{C} 03$ & 3,57 & 2 & 6 & 0,04 \\
\hline $\mathrm{C} 04$ & Emisión de ruidos & 3 & 9 & 0,05 & 5,36 & $\mathrm{C} 04$ & 5,36 & 3 & 9 & 0,05 \\
\hline $\mathrm{C} 05$ & Hablar con los compañeros & 2 & 11 & 0,04 & 3,57 & $\mathrm{C} 05$ & 3,57 & 2 & 11 & 0,04 \\
\hline $\mathrm{C} 06$ & Hacer payasadas & 2 & 13 & 0,04 & 3,57 & $\mathrm{C} 06$ & 3,57 & 2 & 13 & 0,04 \\
\hline $\mathrm{C} 07$ & Realizar acciones Violentas & 3 & 16 & 0,05 & 5,36 & $\mathrm{C} 07$ & 5,36 & 3 & 16 & 0,05 \\
\hline C08 & Utilizar el teléfono móvil & 2 & 18 & 0,04 & 3,57 & $\mathrm{C} 08$ & 3,57 & 2 & 18 & 0,04 \\
\hline $\mathrm{C} 09$ & Desobediencia de normas y autoridad & 3 & 21 & 0,05 & 5,36 & C09 & 5,36 & 3 & 21 & 0,05 \\
\hline C10 & Mofa al profesor & 2 & 23 & 0,04 & 3,57 & $\mathrm{C} 10$ & 3,57 & 2 & 23 & 0,04 \\
\hline C11 & No realiza tareas & 6 & 29 & 0,11 & 10,71 & C11 & 10,71 & 6 & 29 & 0,11 \\
\hline $\mathrm{C} 12$ & $\begin{array}{l}\text { Interrupción cuando los compañeros están } \\
\text { trabajando }\end{array}$ & 2 & 31 & 0,04 & 3,57 & $\mathrm{C} 12$ & 3,57 & 2 & 31 & 0,04 \\
\hline $\mathrm{C} 13$ & Robos y hurtos & 2 & 33 & 0,04 & 3,57 & C13 & 3,57 & 2 & 33 & 0,04 \\
\hline C14 & Hacer ruido en clases & 3 & 36 & 0,05 & 5,36 & C14 & 5,36 & 3 & 36 & 0,05 \\
\hline $\mathrm{C} 15$ & Lenguaje soez & 2 & 38 & 0,04 & 3,57 & C15 & 3,57 & 2 & 38 & 0,04 \\
\hline $\mathrm{C} 16$ & Distracción & 3 & 41 & 0,05 & 5,36 & C16 & 5,36 & 3 & 41 & 0,05 \\
\hline C17 & Interrupción al docente & 2 & 43 & 0,04 & 3,57 & C17 & 3,57 & 2 & 43 & 0,04 \\
\hline C18 & Abandono del aula & 3 & 46 & 0,05 & 5,36 & C18 & 5,36 & 3 & 46 & 0,05 \\
\hline C19 & Pelea y agresiones físicas & 3 & 49 & 0,05 & 5,36 & C10 & 7,14 & 4 & 50 & 0,07 \\
\hline $\mathrm{C} 20$ & Olvido de material & 5 & 54 & 0,09 & 8,93 & $\mathrm{C} 20$ & 7,14 & 4 & 54 & 0,07 \\
\hline $\mathrm{C} 21$ & Mascar chicle & 2 & 56 & 0,04 & 3,57 & C21 & 3,57 & 2 & 56 & 0,04 \\
\hline & Total & 56 & & 1,00 & 100,00 & & 100,00 & 56 & & 1,00 \\
\hline
\end{tabular}




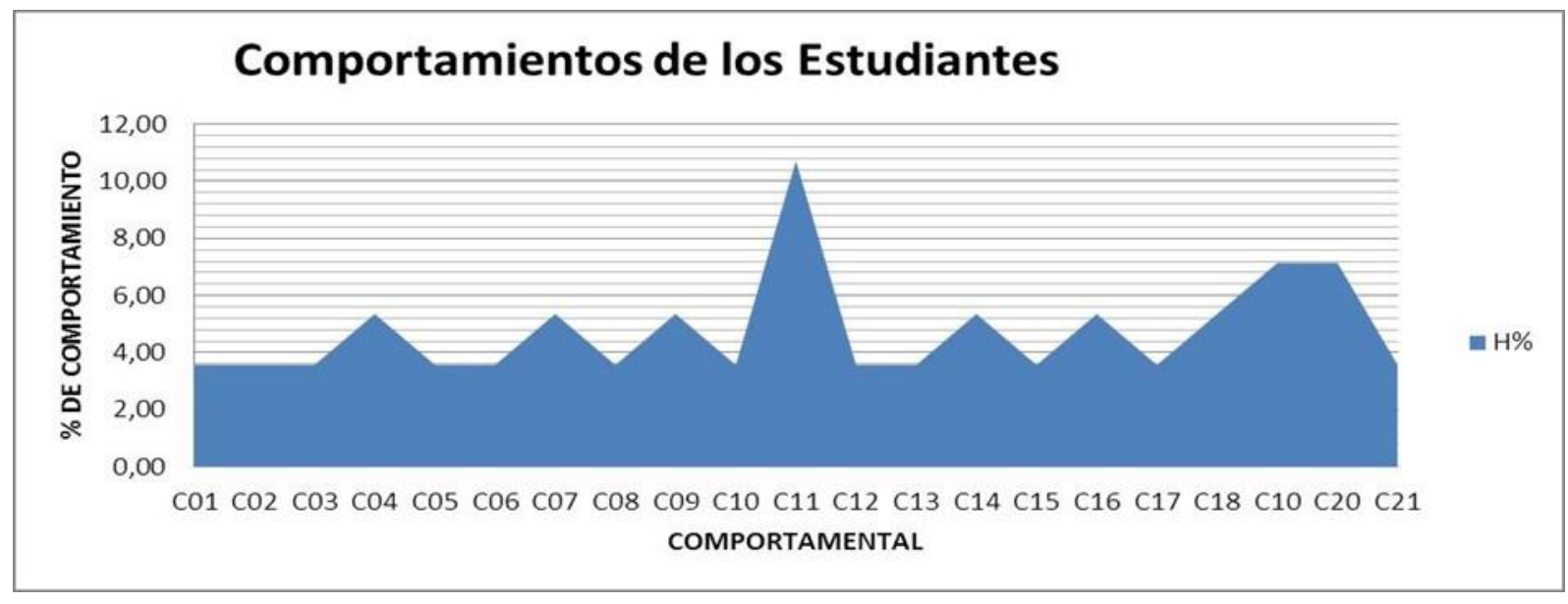

\begin{tabular}{|c|c|c|c|c|c|c|}
\hline \multicolumn{2}{|c|}{$\mathbf{X}$} & $\mathbf{f}$ & $\mathbf{M C}$ & $\mathbf{h} \%$ & $\mathbf{H} \%$ & $\mathbf{F}$ \\
\hline 3 & 5 & 15 & 5,50 & 0,27 & 26,79 & 15 \\
\hline 6 & 8 & 22 & 10,00 & 0,39 & 39,29 & 37 \\
\hline 9 & 11 & 19 & 14,50 & 0,34 & 33,93 & 56 \\
\hline & & 56 & 30,00 & 1,00 & 100,00 & \\
\hline
\end{tabular}

\begin{tabular}{|l|c|c|c|c|c|c|c|c|}
\hline \multicolumn{1}{|c|}{$\mathbf{X}$} & $\mathbf{f ( H )}$ & $\mathbf{f ( M )}$ & $\mathbf{F ( H )}$ & $\mathbf{F}(\mathbf{M})$ & $\mathbf{h}(\mathbf{H})$ & $\mathbf{h}(\mathbf{M})$ & $\mathbf{H}(\mathbf{H}) \mathbf{\%}$ & $\mathbf{H}(\mathbf{M}) \mathbf{\%}$ \\
\hline Inicial 1 & 2 & 2 & 2 & 2 & 0,06 & 0,09 & 5,88 & 9,09 \\
\hline Inicial 2 & 5 & 1 & 7 & 3 & 0,15 & 0,05 & 14,71 & 4,55 \\
\hline Primer año & 3 & 2 & 10 & 5 & 0,09 & 0,09 & 8,82 & 9,09 \\
\hline Segundo & 2 & 3 & 12 & 8 & 0,06 & 0,14 & 5,88 & 13,64 \\
\hline Tercero & 5 & 2 & 17 & 10 & 0,15 & 0,09 & 14,71 & 9,09 \\
\hline Cuarto & 5 & 5 & 22 & 15 & 0,15 & 0,23 & 14,71 & 22,73 \\
\hline Quinto & 2 & 0 & 24 & 15 & 0,06 & 0 & 5,88 & 0,00 \\
\hline Sexto & 5 & 4 & 29 & 19 & 0,15 & 0,18 & 14,71 & 18,18 \\
\hline Septimo & 5 & 3 & 34 & 22 & 0,15 & 0,14 & 14,71 & 13,64 \\
\hline & 34 & 22 & & & 1,00 & 1,00 & 100,00 & 100,00 \\
\hline
\end{tabular}




\section{Discusión}

De los resultados obtenidos se desprenden algunas consideraciones valiosas de naturaleza psicoeducativa. Destaca el relativo nivel de acuerdo o desacuerdo observado entre profesores y los estudiantes sobre la frecuencia de interrupción de comportamientos disruptivos, así como el tipo concreto de conductas que son objeto de sanción; de hecho, no se advierten discrepancias notables, aunque sí diferencias estadísticamente significativas entre lo que unos y otros perciben al respecto.

Acaso sorprende que sean los profesores quienes afirmen intervenir punitivamente en mayor número de ocasiones, frente a una captación sistemáticamente inferior de dichas sanciones por parte del alumnado. Ello significa que una parte nada despreciable de sanciones aplicadas por el profesor no son percibidas por sus alumnos, por lo que cabe esperar que, en el futuro, los alumnos reincidirán en su mal comportamiento, así como el profesor en el suyo, consistente en aplicar una sanción «invisible» a los ojos de sus alumnos.

Si a ello añadimos la existencia de diferencias significativas entre profesores y alumnos en el tipo de sanciones impuestas/percibidas a los distintos comportamientos disruptivos, nos hallamos ante un panorama realmente inquietante, aunque no exento de posibilidades.

Ciertamente, el panorama es inquietante, puesto que a pesar de los esfuerzos que en los últimos tiempos se vienen realizando para mejorar la formación de los docentes en el tema de disciplina escolar, los resultados de nuestra investigación vislumbran un desencuentro persistente entre lo que profesores y estudiantes ven y viven en las aulas. Los profesores afirman incidir sobre los comportamientos disruptivos en el aula mediante unas formas de intervención no reconocidas por los estudiantes, quienes, a su vez, observan acciones por parte de los docentes que según afirman estos últimos no se ajustan a sus patrones de intervención.

\section{Conclusión}

Si realmente se entiende el proceso de enseñanza-aprendizaje con una interacción compleja y multinivel entre quienes participan en el mismo, tamañas divergencias en la forma de captar y entender los intercambios cotidianos por parte de unos y otros constituyen una justificación nada despreciable de no pocas problemáticas que se observan cotidianamente. Entre ellas, sin duda 
alguna, la de los conflictos de disciplina. Cada uno habla su lenguaje y da por sentado que el otro lo comparte; estamos ante una falsa premisa.

Si en párrafos anteriores nos hemos permitido afirmar que, aun tratándose de un panorama inquietante, no andaba exento de posibilidades, es debido a que los resultados de este estudio nos ofrecen, al menos, una plataforma inexplorada desde donde atacar los problemas de disciplina: por una parte, la de lograr una mayor congruencia entre lo que los profesores quieren conseguir nos referimos específicamente al orden y convivencia en clase y lo que hacen para conseguirlo; por otra, entre lo que los profesores hacen para conseguir sus propósitos de disciplina y la manera de comunicarlo a sus alumnos, para así facilitar su identificación y reconocimiento. Sin olvidar que todo ello debe reposar sobre un mejor y más contextualizado conocimiento de lo que los alumnos perciben como sanción y no únicamente sobre lo que los profesores conciben como tal. Puede que éste sí sea un buen comienzo.

\section{Referencias Bibliográficas}

Muy fitness. (2016). Muy fitness. Retrieved from muy fitness: http://muyfitness.com/estilosdisciplina-parental-info_3005/)Abuja, D. (2016). Múltiples problemas de la indisciplina. Quito, Pichincha, Ecuador.

Fuentes, A. S. (2015). educapeques. Retrieved 11 14, 2016, from educapeques:

http://www.educapeques.com/escuela-de-padres/normas-basicas-comportamiento-en-el-aula.html rena.edu.ve. (2012), rena.edu.ve. Retrieved from rena.edu.ve:

http://www.rena.edu.ve/cuartaEtapa/psicologia/Tema15.html

wikipedia.org. (2012). wikipedia.org. Retrieved from wikipedia.org:

http://es.wikipedia.org/wiki/Disciplina_escolar.

Borg, M.G. (1998). Secondary school teachers' perceptions of pupils' un-desirable behaviours.

British Journal of Educational Psychology, 68, 67-79.

Borg, M.G. y Falzon, J.M. (1993). A factor analytic study of teachers' perceptions of pupils' undesirable behaviours: A rejoinder to Langfeld.

British Journal of Educational Psychology, 63, 513-518.

Castelló, A. (2001). Inteligencias. Una integración multidisciplinar. Barcelona: Masson.

Castelló, A. (2002). La inteligencia en acción. Barcelona: Masson. 